\title{
Two-degree-of-freedom Piezoelectric MEMS Energy Harvester Based on Bulk PZT Film
}

\author{
Gang Tang, ${ }^{*}$ Gaoyang Xie, Bo Huang, Bin Xu, \\ Zhibiao Li, Xiaoxiao Yan, Dandan Yuan, and Wenjing Wu \\ Jiangxi Province Key Laboratory of Precision Drive \& Control, Department of Mechanical and Electrical \\ Engineering, Nanchang Institute of Technology, Nanchang 330099, China \\ (Received July 31, 2019; accepted October 10, 2019)
}

Keywords: piezoelectric energy harvester, two-degree-of-freedom, MEMS, bulk PZT film

In this paper, we propose a low-frequency $(<100 \mathrm{~Hz})$ piezoelectric MEMS energy harvester $(\mathrm{PMEH})$ with a two-degree-of-freedom (2DOF) structure to work in two vibration modes for broadband operation. The PMEH is fabricated by bonding a piezoelectric ceramic lead zirconate titanate $\mathrm{Pb}(\mathrm{Zr}, \mathrm{Ti}) \mathrm{O}_{3}(\mathrm{PZT})$ to phosphor bronze, which can work in a high-acceleration ambient with good durability. The maximum open-circuit voltage and output power of the PMEH are $23.6 \mathrm{~V}$ and $64.69 \mu \mathrm{W}$ at the first resonant frequency of $27.4 \mathrm{~Hz}$ when the input vibration acceleration is $2 \mathrm{~g}\left(9.8 \mathrm{~m} / \mathrm{s}^{2}\right)$, while they are $14 \mathrm{~V}$ and $56.75 \mu \mathrm{W}$ at the second resonant frequency of $93.4 \mathrm{~Hz}$ when the input vibration acceleration is $8 \mathrm{~g}$, respectively. The fabricated PMEH scavenged the vibration energy of a vacuum compression pump and an ultrasonic cleaner and generated maximum output powers of 10.45 and $16.59 \mu \mathrm{W}$, respectively. The experimental results show that the device has good environmental adaptability.

\section{Introduction}

Energy harvesting plays an important role in the field of low-power energy supplies, such as wireless sensors and microactuators, especially in the medical, communications, and military fields. ${ }^{(1)}$ Among the environmental energy sources, mechanical vibration energy is the most ubiquitous and can be found everywhere in our daily lives. Therefore, various harvesters have been proposed for converting waste ambient mechanical energy into electrical energy using different mechanisms, such as electrostatic, ${ }^{(2,3)}$ electromagnetic, ${ }^{(4,5)}$ triboelectric, ${ }^{(6,7)}$ and piezoelectric mechanisms. ${ }^{(8,9)}$ Owing to their simple configuration, high energy density, and compatibility with MEMS processes, piezoelectric MEMS energy harvesters (PMEHs) have been intensively developed. The coupling coefficient of the piezoelectric material plays an important role in PMEHs, which directly affects the energy conversion efficiency. Generally, the performance of a PMEH is highly dependent on the piezoelectric properties of the material. Piezoelectric ceramic lead zirconate titanate (PZT) is a suitable material for PMEHs because it has excellent piezoelectric properties, and most PMEHs are formed using PZT together with a

*Corresponding author: e-mail: tanggang@nit.edu.cn https://doi.org/10.18494/SAM.2019.2543 
silicon substrate. Well-crystallized PZT thin films with a thickness of $\mu \mathrm{m}$ order or less can be formed by the sol-gel method, sputtering, or pulse laser deposition. However, the PMEHs using PZT thin films exhibit a low output power on the order of $\mu \mathrm{W}$ because of the limited thickness of the piezoelectric films. ${ }^{(10,11)}$ Although the hydrothermal method ${ }^{(12)}$ and screen printing process $^{(13)}$ have been developed for thick films over $5 \mu \mathrm{m}$ in thickness, a sintering temperature of more than $550{ }^{\circ} \mathrm{C}$ is required to provide a high piezoelectricity, which limits the application of the subsequent MEMS fabrication process. To obtain a sufficiently powerful PMEH, bulk PZT bonding and thinning techniques have been developed. ${ }^{(14-17)}$ In our previous work, we reported a PMEH with a $14-\mu$ m-thick bulk PZT film based on a silicon substrate, which was realized by low-temperature $\left(175^{\circ} \mathrm{C}\right)$ bonding and thinning techniques. ${ }^{(14)}$ Because silicon is relatively brittle and not suitable for high-vibration-acceleration applications, in later research, we proposed a PMEH based on PZT and phosphor bronze bonding that could work at low frequencies and high acceleration. ${ }^{(15-17)}$ The experimental results for the devices mentioned above show that a thinned bulk PZT film can have both good density and an appropriate thickness, and the fabricated PMEHs using thinned bulk PZT films exhibited a high output performance.

On the other hand, a vibration-based PMEH is often designed as a single-degree-offreedom (1DOF) system, and one of the major problems of 1DOF PMEHs is a narrow operating bandwidth, which severely limits the performance of the PMEH considering the wide bandwidth of ambient vibrations. To overcome this critical issue, many approaches to broadening the operating bandwidth of piezoelectric energy harvesters, such as nonlinear techniques ${ }^{(18,19)}$ and multimodal harvesting techniques, ${ }^{(20-23)}$ have been proposed. Multimodal energy harvesters are considered more efficient when matching multiple frequencies to better utilize kinetic energy in these scenarios. For PMEHs, multimodal techniques are also more advantageous in terms of wafer-level fabrication and minimal post-assembly processes. ${ }^{(24)}$

In this study, we have developed a PMEH with a two-degree-of-freedom (2DOF) multimodal structure based on a bulk PZT film, which can work at two low resonant frequencies $(<100 \mathrm{~Hz})$ and scavenge the vibration energy at a high acceleration of $8.0 \mathrm{~g}$. The PMEH was fabricated by thinning bulk PZT and bonding on a flexible phosphor bronze substrate at a low temperature. The structural design, MEMS fabrication, and characterization of the PMEH were demonstrated in detail. Moreover, the application testing shows that the PMEH can effectively scavenge the vibration energy from a vacuum pump and an ultrasonic cleaner at the first and second resonance frequencies, respectively.

\section{Harvester Design}

The schematic structure of the proposed 2DOF PMEH is shown in Fig. 1(a). Although a similar configuration has been well developed in large-scale devices, ${ }^{(22)}$ the fabrication of a microscale generator is still difficult. To increase the output power of the PMEH and enable it to operate in low-frequency and high-acceleration environments, a thinned bulk PZT film is fabricated on the phosphor bronze substrate of the PMEH. The proposed PMEH comprises one main cantilever beam and an inner secondary cantilever beam, and two tungsten proof masses 


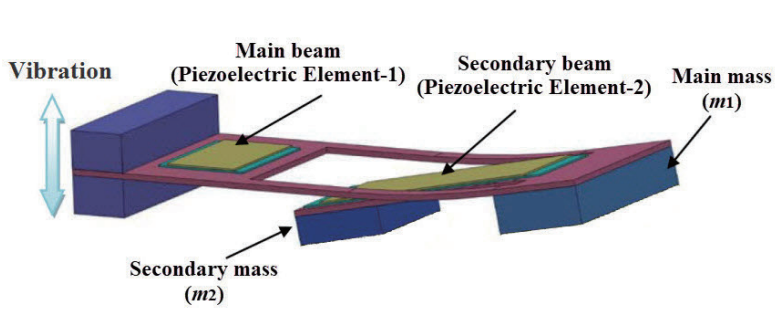

(a)

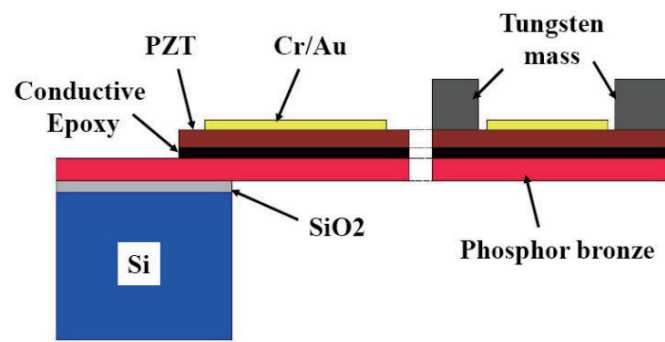

(b)

Fig. 1. (Color online) (a) Schematic of 2DOF PMEH and (b) lay sequence of the designed PMEH.

at the tips of the main and secondary cantilever beams. Both the main and secondary cantilever beams have multilayer structures, as shown in Fig. 1(b). The multilayer beams are made up of a top electrode $(\mathrm{Cr} / \mathrm{Au})$, a PZT thick film, an epoxy resin, and a phosphor bronze substrate.

The lumped-mass model of the proposed 2DOF PMEH is shown in Fig. 2. In this work, our goal is to design a structure to provide two low-frequency resonant peaks so that the harvester is more robust in practical vibration environments. The main subsystem of the 2DOF PMEH has the main mass $m_{1}$, mechanical damping $\eta_{1}$, and spring stiffness $k_{1}$, and the first piezoelectric element inserted between the base and $m_{1}\left(C_{p 1}\right.$ is its clamped capacitance). The secondary subsystem has the secondary mass $m_{2}$ and spring stiffness $k_{2}$, and the second piezoelectric element inserted between the masses $m_{1}$ and $m_{2}\left(C_{p 2}\right.$ is its clamped capacitance). The governing equation of the proposed 2DOF PMEH system is derived as ${ }^{(25)}$

$$
\left\{\begin{array}{l}
m_{1} \cdot \ddot{u}_{1}+\eta_{1} \cdot\left(\dot{u}_{1}-\dot{u}_{0}\right)+\eta_{2}\left(\dot{u}_{1}-\dot{u}_{2}\right)+k_{1} \cdot\left(u_{1}-u_{0}\right)+k_{2} \cdot\left(u_{1}-u_{2}\right)+\alpha_{1} V_{1}-\alpha_{2} V_{2}=0 \\
m_{2} \cdot \ddot{u}_{2}+\eta_{2} \cdot\left(\dot{u}_{2}-\dot{u}_{1}\right)+k_{2} \cdot\left(u_{2}-u_{1}\right)+\alpha_{2} V=0 \\
\frac{V_{1}}{R_{1}}+C_{p 1} \cdot V_{1}-\alpha_{1} \cdot\left(\dot{u}_{1}-\dot{u}_{0}\right)=0 \\
\frac{V_{2}}{R_{2}}+C_{p 2} \cdot V_{2}-\alpha_{2} \cdot\left(\dot{u}_{2}-\dot{u}_{1}\right)=0
\end{array}\right.
$$

where $u_{0}, u_{1}$, and $u_{2}$ are the displacements of the base, mass $m_{1}$, and mass $m_{2} ; \alpha_{1}$ and $\alpha_{2}$ are the electromechanical coupling coefficients of the first and second piezoelectric elements; $R_{1}$ and $R_{2}$ are the load resistances of the two piezoelectric elements; and $V_{1}$ and $V_{2}$ are the voltages across $R_{1}$ and $R_{2}$, respectively.

Using Eq. (1), the voltages $V_{1}$ and $V_{2}$ have been solved, and the relationship between the two resonant frequencies has already been determined. ${ }^{(25)}$ The parameters affecting the performance of the proposed PMEH include the coupling strength of the first and second piezoelectric elements, the mass ratio $\mathrm{m}_{1} / \mathrm{m}_{2}$, the stiffness ratio $k_{1} / k_{2}$, and the damping ratios $\eta_{1} / 2 \sqrt{k_{1} \cdot m_{1}}$ and $\eta_{2} / 2 \sqrt{k_{2} \cdot m_{2}}$ of the main and secondary subsystems of the PMEH, respectively. These form a theoretical foundation for the proposed 2DOF PMEH design. The details of the design parameters of the proposed 2DOF PMEH are listed in Table 1. 


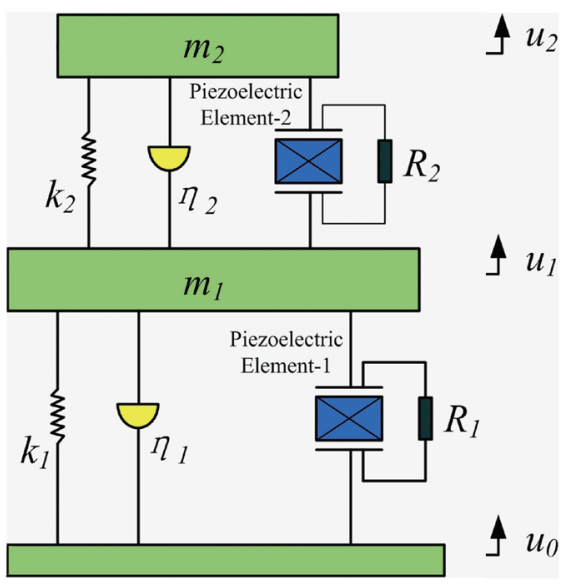

Fig. 2. (Color online) Lumped-mass model of proposed 2DOF PMEH with bulk PZT thick film.
Table 1

Main design parameters of proposed 2DOF PMEH.

\begin{tabular}{lcc}
\hline Component & Design parameter & Value \\
\hline \multirow{3}{*}{ Main beam } & Length & $27 \mathrm{~mm}$ \\
& Width & $8 \mathrm{~mm}$ \\
& Height & $103.2 \mu \mathrm{m}$ \\
\hline \multirow{3}{*}{ Secondary beam } & Length & $8 \mathrm{~mm}$ \\
& Width & $3.2 \mathrm{~mm}$ \\
& Height & $103.2 \mu \mathrm{m}$ \\
\hline \multirow{3}{*}{ Main mass } & Length & $3 \mathrm{~mm}$ \\
& Width & $7 \mathrm{~mm}$ \\
& Height & $1.5 \mathrm{~mm}$ \\
\hline \multirow{3}{*}{ Secondary mass } & Length & $1.5 \mathrm{~mm}$ \\
& Width & $3 \mathrm{~mm}$ \\
& Height & $1.5 \mathrm{~mm}$ \\
\hline \multirow{3}{*}{ PZT thick films } & PZT thickness & $50 \mu \mathrm{m}$ \\
& Bop Cr/Au thickness & $0.2 \mu \mathrm{m}$ \\
& Bottom conductive & $3 \mu \mathrm{m}$ \\
\hline Phosphor bronze & epoxy thickness & $50 \mu \mathrm{m}$ \\
\hline PMEH & Thickness & $60.54 \mathrm{~mm}$ \\
\hline
\end{tabular}

The resonant frequency of the designed PMEH was simulated by ANSYS software. The first two vibration modes are predicted as shown in Fig. 3. The resonant frequencies from the first and second modes are 33.463 and $98.294 \mathrm{~Hz}$, respectively, which are less than $100 \mathrm{~Hz}$, meeting the design requirement of less than $100 \mathrm{~Hz}$. The first mode shape is the normal mode in which the main beam vibrates perpendicular to the plane of the system, and the second mode shape is also the normal mode in which the secondary beam vibrates perpendicular to the plane of the system. In the first mode, the maximum displacement occurs at the free end of the main beam and the corresponding stress concentrates on the main beam, whereas in the second mode, the maximum displacement occurs at the free end of the secondary beam and the corresponding stress concentrates on the secondary beam. This indicates that the piezoelectric element from the main beam can operate effectively at the first response frequency, whereas that from the secondary beam can operate effectively at the second response frequency.

\section{Fabrication of PMEH}

As mentioned above, the structure of the 2DOF PMEH is two PZT/phosphor bronze composite beams with two tungsten masses on their free ends. The techniques of microfabrication mainly involve bulk PZT film preparation on phosphor bronze, bulk silicon micromachining, laser cutting and patterning, and mass assembly. The basic fabrication process for the PMEH is shown in Fig. 4.

The fabrication process began with a $450-\mu$ m-thick bare silicon wafer substrate, on which a $2-\mu \mathrm{m}$-thick $\mathrm{SiO}_{2}$ layer was grown on one side as an electrical isolation layer by plasmaenhanced chemical vapor deposition (PECVD) [Fig. 4(a)]. Then, a 60- $\mu$ m-thick phosphor bronze layer and the prepared silicon wafer were bonded together facing each other. To acquire 


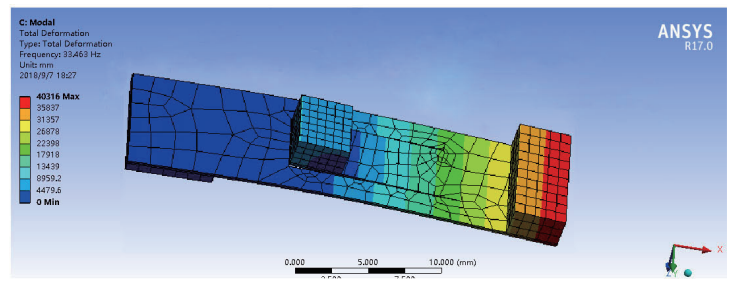

(a)

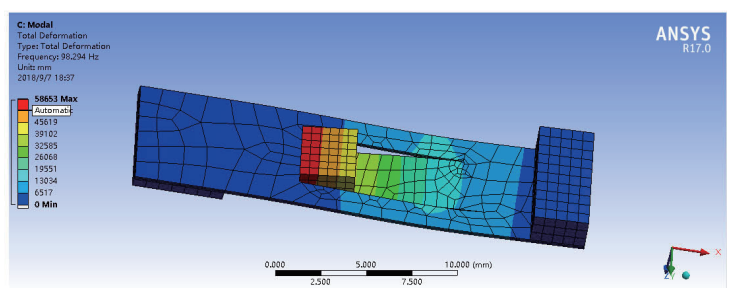

(c)

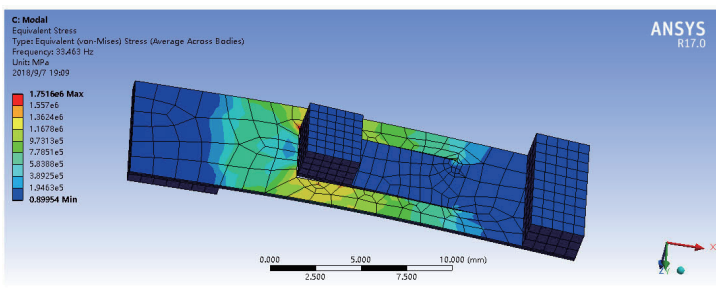

(b)

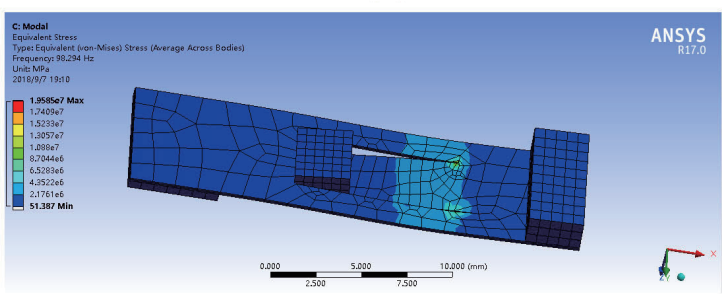

(d)

Fig. 3. (Color online) Displacement (a) and stress (b) distributions in the first mode, and the displacement (c) and stress (d) distributions in the second mode.

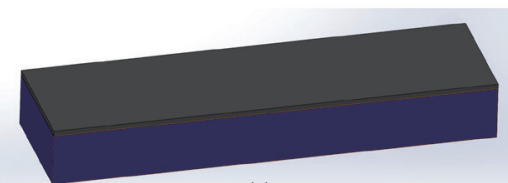

(a)

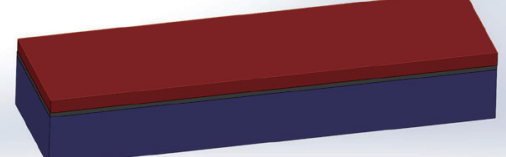

(b)

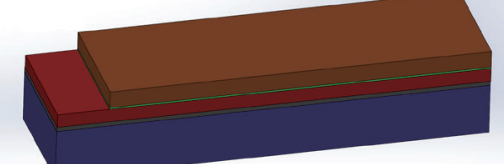

(c)

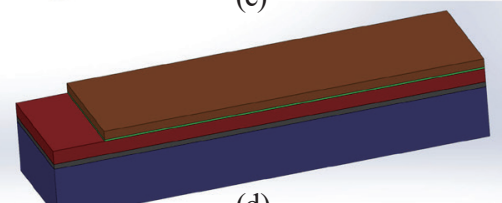

(d)

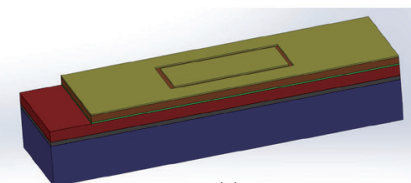

(e)

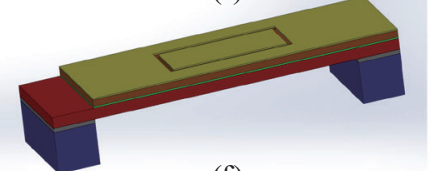

(f)

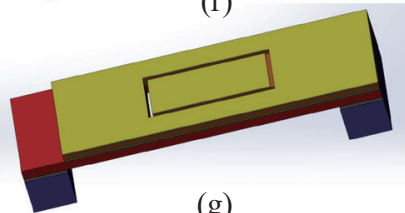

(g)

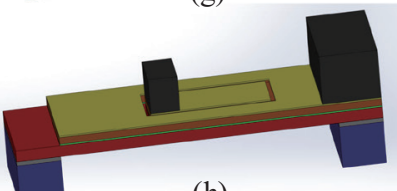

(h)

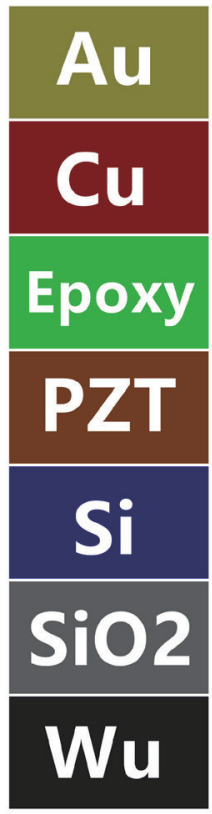

Fig. 4. (Color online) Fabrication process flow of the designed PMEH.

the design parameters and improve the adhesion strength between bulk PZT and phosphor bronze in the following process, the phosphor bronze was thinned and polished by mechanical lapping [Fig. 4(b)]. Thereafter, one layer of conductive epoxy resin was screen-printed as an intermediate layer on the polished surface of the phosphor bronze. Then, surface-polished bulk PZT with a thickness of $400 \mu \mathrm{m}$ and the prepared phosphor bronze were bonded together in a vacuum oven [Fig. 4(c)]. To achieve a sufficient bonding strength, the following bonding parameters and steps must be strictly implemented. A bonding pressure of $0.1 \mathrm{MPa}$ was 
applied through a laboratory-built force device and the bonding temperature was set from 60 to $175^{\circ} \mathrm{C}$ with the temperature raised by $15^{\circ} \mathrm{C}$ every $10 \mathrm{~min}$ and held for more than $1 \mathrm{~h}$ at $175{ }^{\circ} \mathrm{C}$. After completing the bonding of PZT and phosphor bronze, the bulk PZT layer was thinned to the design thickness of about $50 \mu \mathrm{m}$ by mechanical lapping to maintain good uniformity and flexibility [Fig. 4(d)]. Following that, a Cr/Au layer with a thickness of $200 \mathrm{~nm}$ as the top electrode was sputtered on the thinned bulk PZT layer. The sputtered top electrode was then patterned and etched through lithography and ion beam milling [Fig. 4(e)]. The handle Si layer was etched from the back side to form a cantilever with a silicon proof mass structure by deep reactive ion etching (DRIE) [Fig. 4(f)]. Then, an inner secondary cantilever structure was patterned by ultraviolet laser cutting [Fig. 4(g)]. Finally, two rectangular tungsten proof masses were fabricated by dicing and assembled on the free ends of the main and secondary beams [Fig. 4(h)]. The addition of the two masses can reduce the first and second resonant frequencies of this device.

A cross-sectional SEM image of the fabricated PMEH is shown in Fig. 5(a). The PZT/epoxy/ phosphor bronze layers can be seen clearly, and it is found that the thicknesses of the thinned bulk PZT film, epoxy layer, and phosphor bronze supporting layer are about 49, 2, and $50 \mu \mathrm{m}$, respectively. Figure 5(b) shows a SEM image of the polished bulk PZT. The surface of the polished bulk PZT is smooth, but some scattered pores and some faults exist on the surface. The pores are usually inevitable in bulk PZT materials because of the high-temperature sintering process, although the faults can be produced by lapping. ${ }^{(14)}$ An optical image of the fabricated prototype assembled with printed circuit board (PCB) is shown in Fig. 5(c). From the image, we can clearly see the 2DOF structure with two cantilever beams and two masses.

\section{Testing and Discussion}

\subsection{Experimental testing setup}

An impedance analyzer (TZDM-200-300) was used to evaluate the impedance and phase angle of the fabricated PMEH versus the excitation frequency. The output performance of the

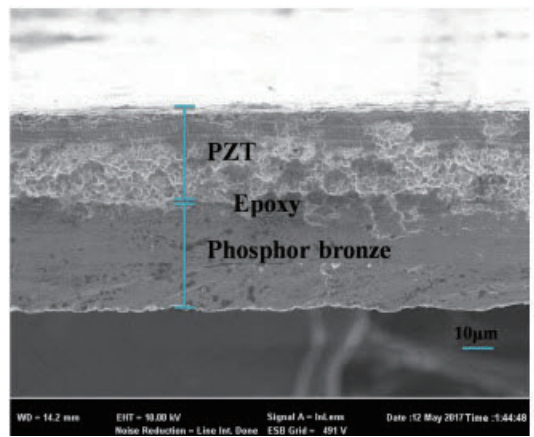

(a)

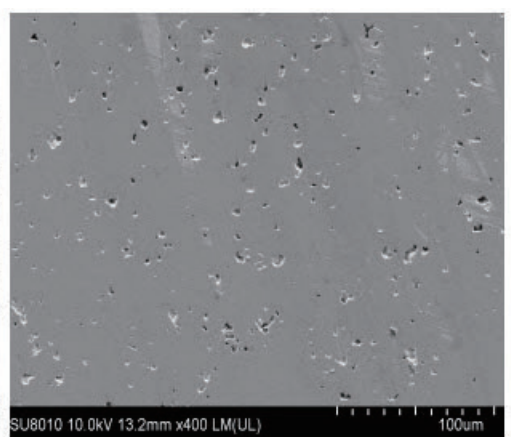

(b)

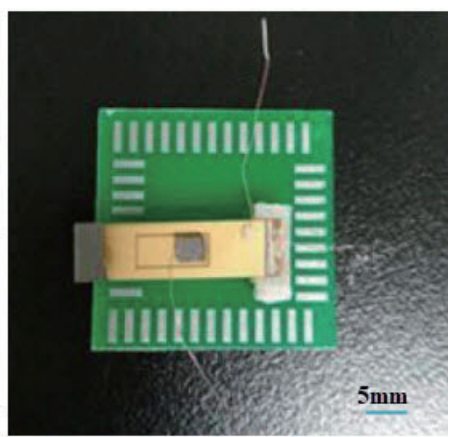

(c)

Fig. 5. (Color online) (a) Cross-sectional SEM image of thinned bulk PZT layer bonded with phosphor bronze supporting layer, (b) SEM image of polished bulk PZT, and (c) optical image of the fabricated PMEH assembled with PCB. 
prototype was measured using a vibration-testing platform including a vibrator (JZK-5), power amplifier (YE5971A), a waveform generator (33205A), an acceleration monitor (YE5932B), a resistance box (ZX99-1A), and an oscilloscope (MDO3000), as shown in Fig. 6. During the testing, the fabricated PMEH device was mounted on the vibrator. The waveform generator and power amplifier were used to drive the vibrator and regulate the vibration strength, respectively. The vibration strength was monitored by the acceleration monitor and the electrical output of the device was monitored by the oscilloscope.

\subsection{Output performance}

Figure 7 shows the measured impedance of the fabricated PMEH. The testing result indicated that the resonant frequencies of the main and secondary beams are 28 and $98 \mathrm{~Hz}$, respectively, which have good agreement with the first and second resonant frequencies acquired by FEM simulation.

The open-circuit voltage from the main beam of the PMEH versus the excitation vibration frequency was measured under different vibration accelerations [Fig. 8(a)]. It can be seen that at the vibration accelerations of $0.5,1,1.5$, and $2 \mathrm{~g}\left(9.8 \mathrm{~m} / \mathrm{s}^{2}\right)$, the resonant frequency of the main

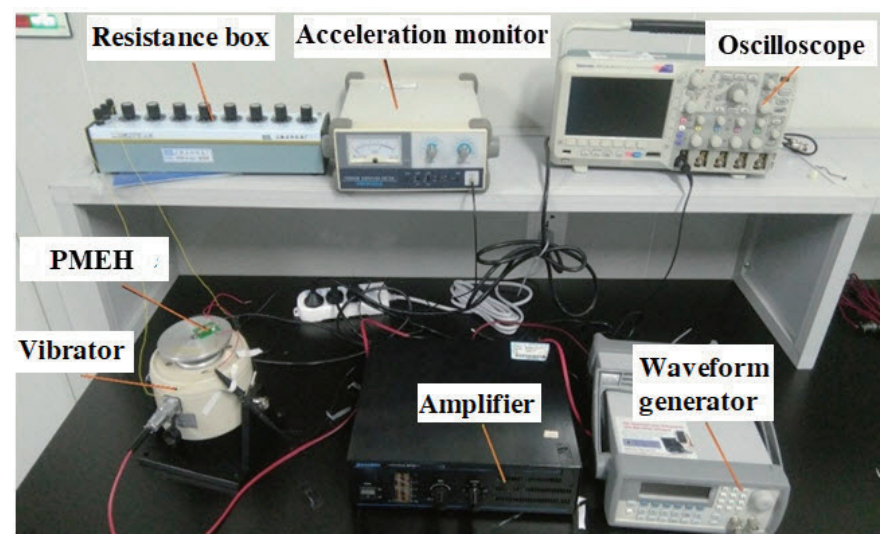

Fig. 6. (Color online) Testing setup used in this study.

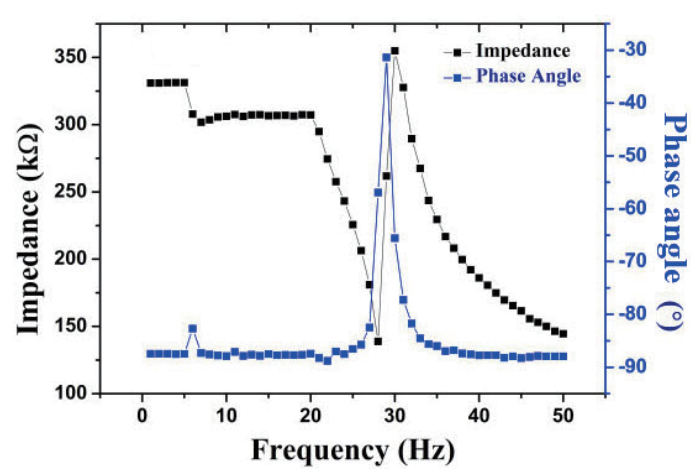

(a)

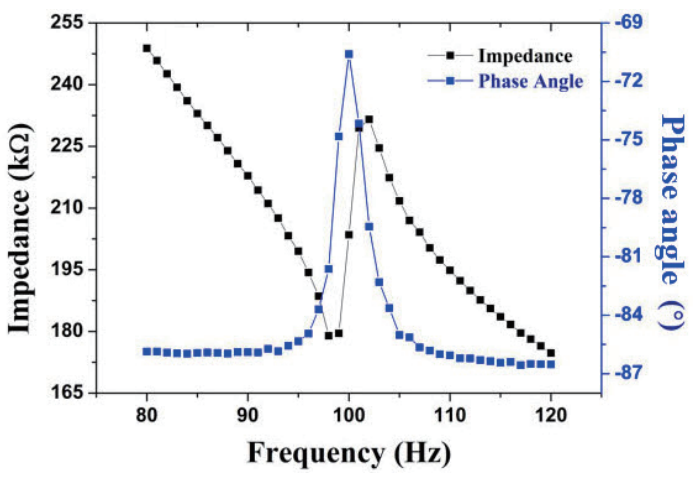

(b)

Fig. 7. (Color online) Impedance testing result from the (a) main and (b) secondary beams of the PMEH. 

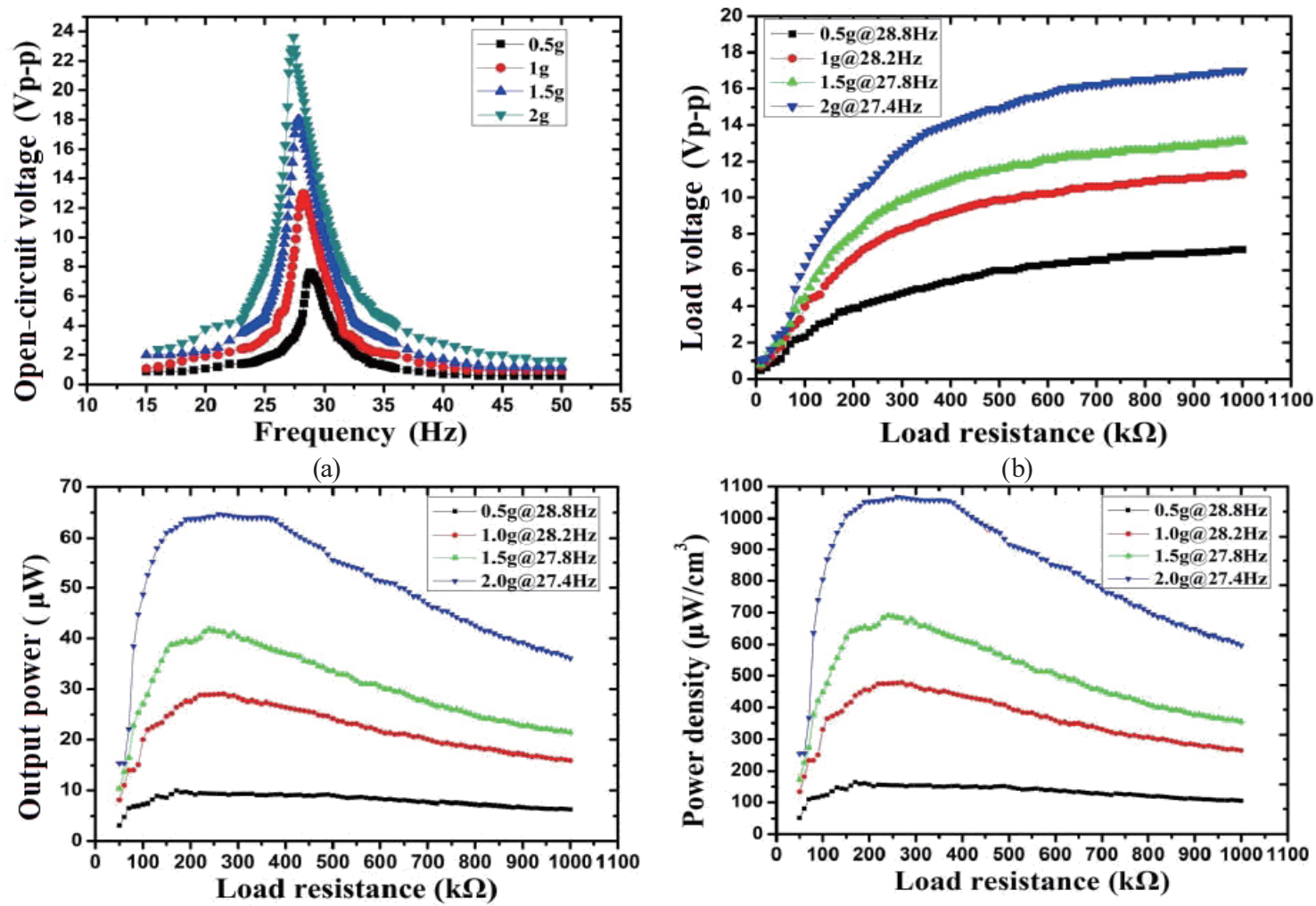

(c)

(d)

Fig. 8. (Color online) Output performance characteristics from the main beam of the fabricated PMEH: (a) open-circuit voltage, (b) load voltage, (c) output power, and (d) power density delivered to the load in the first mode of the PMEH.

beam, i.e., the first resonant frequency of the PMEH, is determined to be $28.8,28.2,27.8$, and $27.4 \mathrm{~Hz}$, respectively. The measured resonant frequency gradually decreases with increasing vibration acceleration, which is caused by the nonlinear change in the Young's modulus of PZT. ${ }^{(25)}$ The maximum open-circuit output voltage increases with the vibration acceleration. When the vibration acceleration increases from 0.5 to $2.0 \mathrm{~g}$, the maximum peak-peak voltage under the open-circuit condition is determined to be $7.6,13,18$, and $23.6 \mathrm{~V}$ at the above-mentioned four vibration accelerations, respectively.

Figure 8(b) shows the output load voltage from the main beam at different load resistances under different vibration accelerations from 0.5 to $2.0 \mathrm{~g}$. The output voltage gradually increases with the external impedance. However, the rate of increase at low impedances is greater than that at high impedances.

From the load output voltage, the corresponding average output power can be calculated as

$$
P=\frac{\left(\frac{V_{p-p}}{2 \sqrt{2}}\right)^{2}}{R},
$$

where $V_{p-p}$ is the peak-peak load voltage and $R$ is the load resistance. 
The output power of the main beam obtained from Eq. (2) versus load resistance under different vibration accelerations at the resonant frequencies is shown in Fig. 8(c). With increasing load resistance, the output power first increases and then decreases at each acceleration. There is a peak value of the output power at the optimal matched resistance of $260 \mathrm{k} \Omega$. As shown in Fig. 8(c), the maximum output power is $64.69 \mu \mathrm{W}$ at the vibration acceleration of $2 \mathrm{~g}$. Moreover, the output power density of the fabricated PMEH at the first resonant frequency can be defined as the output power of the main beam divided by the effective volume of the PMEH, as shown in Fig. 8(d). The optimal output power density of the fabricated PMEH in the first mode is $1068.55 \mu \mathrm{W} / \mathrm{cm}^{3}$ at the vibration acceleration of $2.0 \mathrm{~g}$ and the resonant frequency of $27.4 \mathrm{~Hz}$.

As mentioned above, the secondary beam of the fabricated PMEH plays a major role in the second mode of the PMEH. The open-circuit voltage versus the excited vibration frequency, and the load voltage, output power, and power density versus the load resistance for accelerations from 1 to $8 \mathrm{~g}$ are shown in Figs. 9(a)-9(d), respectively. The resonant frequency in the second mode of the PMEH at accelerations of $1,2,3,4,5,6,7$, and $8 \mathrm{~g}$ is determined to be 101.3, 99.9, $99,98.2,96.8,95.3,94.3$, and $93.4 \mathrm{~Hz}$, respectively. The corresponding maximum open-circuit

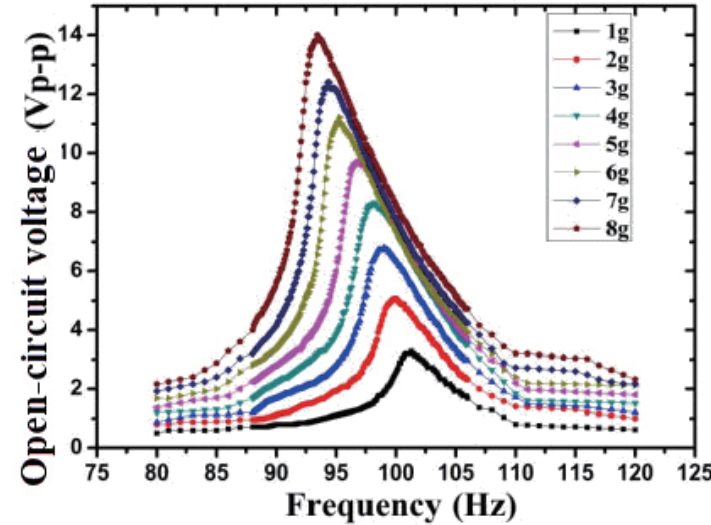

(a)

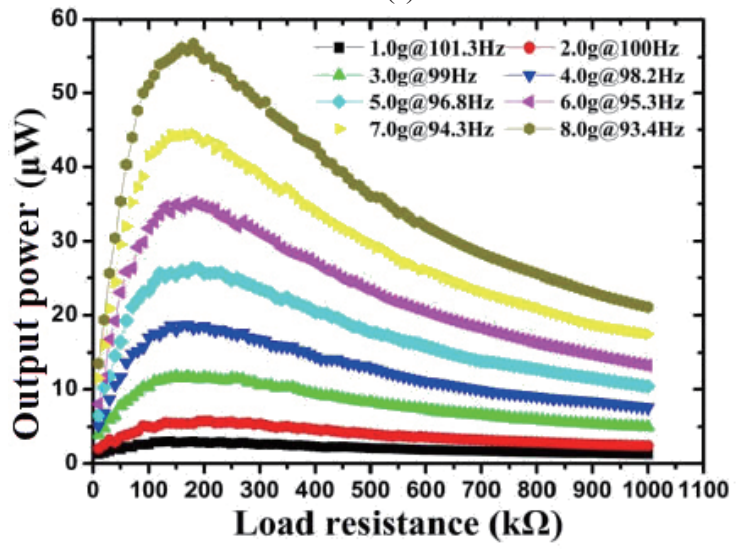

(c)

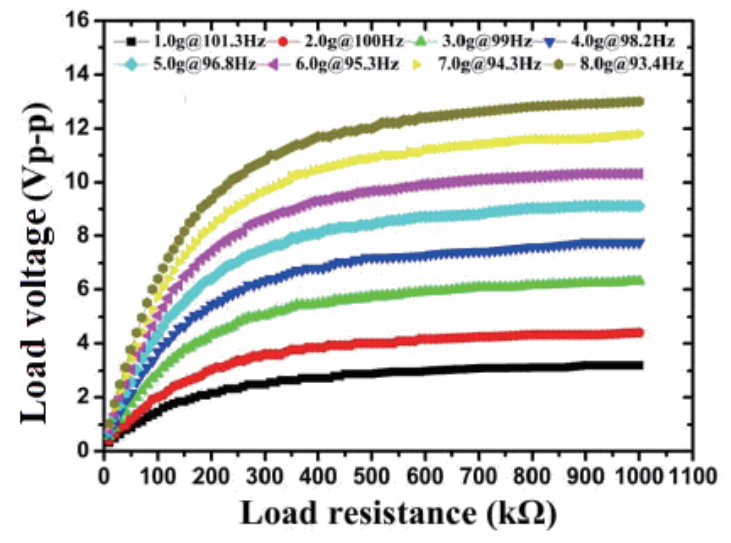

(b)

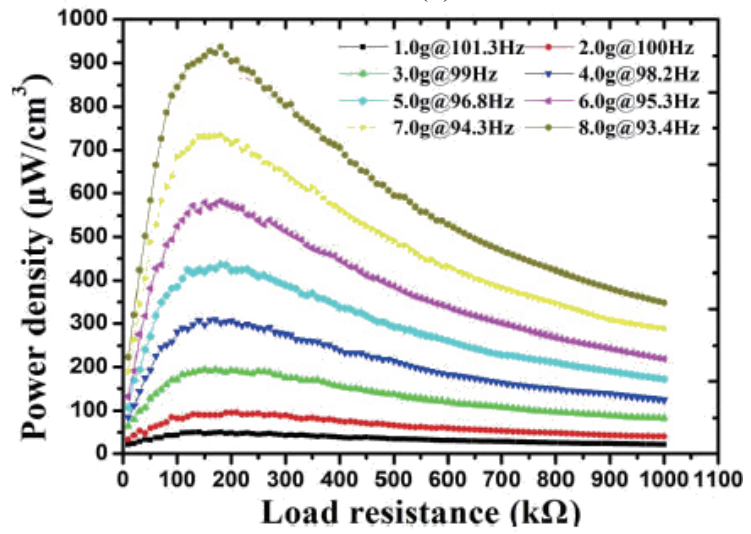

(d)

Fig. 9. (Color online) Output performance characteristics from the secondary beam of the fabricated PMEH: (a) open-circuit voltage, (b) load voltage, (c) output power, and (d) power density delivered to the load in the second mode of the PMEH. 
voltages are $3.28,5.06,6.8,8.32,9.72,11.2,12.4$, and $14 \mathrm{~V}$, respectively. In addition, the optimal resistance is $180 \mathrm{k} \Omega$ and the maximum output powers are $2.98,5.54,11.78,18.68,26.35,33.99$, 44.18 , and $56.75 \mu \mathrm{W}$ when the load resistance is matched with the optimal resistance of $180 \mathrm{k} \Omega$ at the above-mentioned accelerations, respectively. Moreover, the optimal output power density of the fabricated PMEH in the second mode is $937.40 \mu \mathrm{W} / \mathrm{cm}^{3}$ at the vibration acceleration of $8.0 \mathrm{~g}$ and the resonant frequency of $93.4 \mathrm{~Hz}$. This indicates that the power density of the PMEH reported in this paper demonstrates comparable performance characteristics for two working modes at low frequencies.

\subsection{Applications of the PMEH prototype}

To verify the capacity of the fabricated PMEH in practical applications, the PMEH was tested on a vacuum compression pump and an ultrasonic cleaner. Figures 10(a) and 11(a) show the photographs of the fabricated PMEH fixed on the pump and cleaner, respectively. The

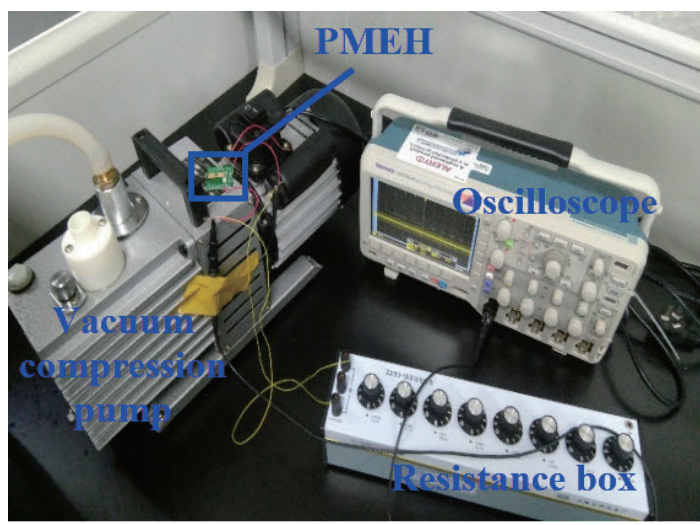

(a)

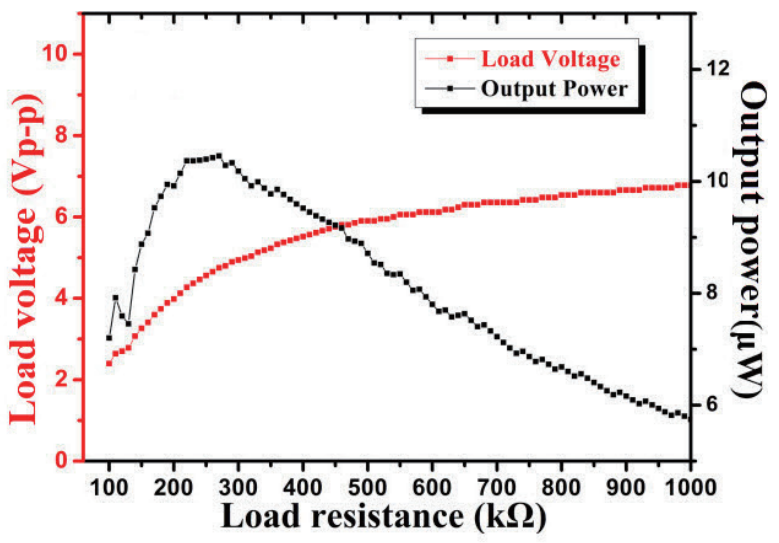

(b)

Fig. 10. (Color online) Testing of output from the main beam of the fabricated PMEH on the vacuum compression pump: (a) testing setup and (b) output load voltage and power delivered to the load versus resistance.

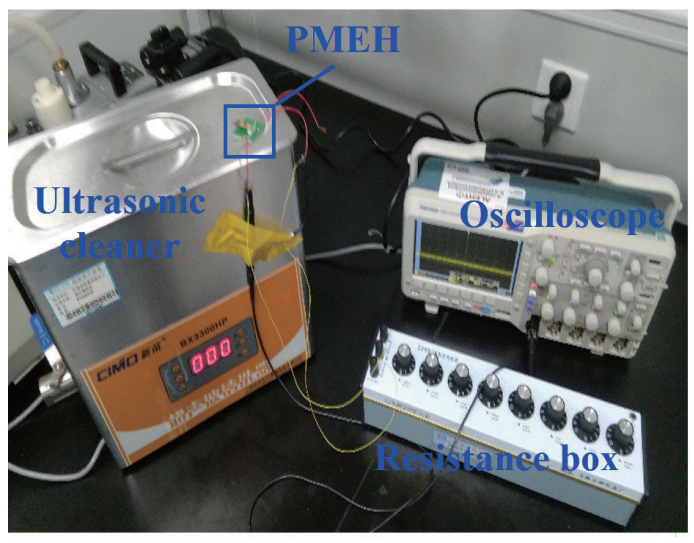

(a)

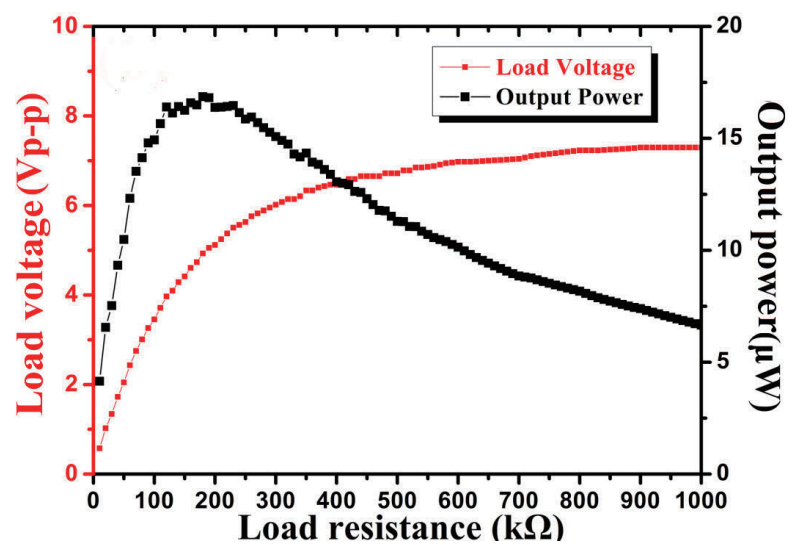

(b)

Fig. 11. (Color online) Testing of output from the secondary beam of the fabricated PMEH on the ultrasonic cleaner: (a) testing setup and (b) the output load voltage and power delivered to the load versus resistance. 
resistance box connected to the top and bottom electrodes of the fabricated PMEH provides variable resistance and the oscilloscope is used to record the output voltage of the PMEH. When the vacuum pump or ultrasonic cleaner is in operation, the vibration of the pump and cleaner drives the oscillation of the main or secondary beam of the manufactured PMEH. According to the test results, the main beam of the PMEH can work effectively under the vibration of the pump, while the secondary beam can work effectively under the vibration of the cleaner. The obtained output load (peak-peak) voltages and output powers from the main and secondary beams of the fabricated PMEH at different load resistances are shown in Figs. 10(b) and 11(b), respectively. When the external resistance is $1 \mathrm{M} \Omega$, the load voltages output from the main and secondary beams reach 6.78 and $7.296 \mathrm{~V}$, respectively. In addition, we find that the maximum output powers from the main and secondary beams are 10.45 and $16.59 \mu \mathrm{W}$, respectively. At these maximum output powers, the corresponding output voltages from the main and secondary beams are 4.752 and $4.928 \mathrm{~V}$, respectively, which are higher than the minimum voltage required for the rectifier circuit diode band gaps. Considering the rapid development of low-power microelectronic devices such as many commercial sensors, the power consumption of these devices is very low, even on the order of $\mu \mathrm{W}$ with operating voltages below $3 \mathrm{~V}$ in the inactive mode. Therefore, the PMEH can be used to provide power for some electronic devices and systems with low power consumption.

\section{Conclusions}

We have demonstrated the design, fabrication, and characterization of a 2DOF PMEH that can operate at two low resonant frequencies and high acceleration. The harvester comprises a main beam and an inner secondary beam, which can effectively operate at the first and second resonant frequencies, respectively. The open-circuit (peak-peak) voltage, the maximum output power, and the corresponding power density from the main beam of the PMEH at the input vibration acceleration of $2 \mathrm{~g}$ and the resonant frequency of $27.4 \mathrm{~Hz}$ are $23.6 \mathrm{~V}, 64.69 \mu \mathrm{W}$, and $1068.55 \mu \mathrm{W} / \mathrm{cm}^{3}$, respectively. On the other hand, when the input vibration acceleration is $8 \mathrm{~g}$, the open-circuit voltage, the maximum output power, and the corresponding power density from the secondary beam of the PMEH at the resonant frequency of $93.4 \mathrm{~Hz}$ are $14 \mathrm{~V}, 56.75 \mu \mathrm{W}$, and $937.40 \mu \mathrm{W} / \mathrm{cm}^{3}$, respectively. Moreover, experiments on the application of the PMEH, in which the vibration energy of a vacuum compression pump and an ultrasonic cleaner was converted into electric energy, were performed. The output power from the main beam when the fabricated PMEH operates on the vacuum pump is $10.45 \mu \mathrm{W}$, while that from the secondary beam is $16.59 \mu \mathrm{W}$ when the PMEH operates on the ultrasonic cleaner. The experimental results show that the PMEH can power low-power electronic devices and systems in wireless sensor networks.

\section{Acknowledgments}

This work was supported by the National Natural Science Foundation of China (51565038) and the Science and Technology Project of Jiangxi Provincial Education Department (GJJ170986 and GJJ180938). 


\section{References}

1 G. Tang, B. Bao, Z. Yi, G. Li, G. Li, B. Yang, and J. Liu: Sens. Mater. 29 (2017) 1733. https://doi.org/10.18494/ SAM.2017.1727

2 Y. Zhang, T. Wang, A. Luo, Y. Hu, X. Li, and F. Wang: Appl. Energy 212 (2018) 362. https://doi.org/10.1016/ j.apenergy.2017.12.053

3 K. Tao, L. Tang, J. Wu, S. W. Lye, H. Chang, and J. Miao: J. Microelectromech. Syst. 27 (2018) 276. https://doi. org/10.1109/JMEMS.2018.2792686

4 Y. Pan, T. Lin, F. Qian, C. Liu, J. Yu, J. Zuo, and L. Zuo: Appl. Energy 247 (2019) 309. https://doi.org/10.1016/ j.apenergy.2019.03.051

5 T. Lin, J. J. Wang, and L. Zuo: Mechatronics 53 (2018) 277. https://doi.org/10.1016/j.mechatronics.2018.06.019

6 R. K. Gupta, Q. Shi, L. Dhakar, T. Wang, C. H. Heng, and C. Lee: Sci. Rep. 7 (2017) 1. https://doi.org/10.1038/ srep41396

7 L. Dhakar, F. E. H. Tay, and C. Lee: J. Micromech. Microeng. 24 (2014) 1. https://doi.org/10.1088/0960$1317 / 24 / 10 / 104002$

8 G. Li, Z. Yi, Y. Hu, J. Liu, and B. Yang: J. Micromech. Microeng. 28 (2018) 1. https://doi.org/10.1088/13616439/aac58a

9 G. Tang, B. Yang, J. Q. Liu, B. Xu, H. Y. Zhu, and C. S. Yang: Sens. Actuators, A 205 (2014) 150. https://doi. org/10.1016/j.sna.2013.11.007

10 D. N. Shen, J. H. Park, J. H. Noh, S. Y. Choe, S. H. Kim, H. C. Wikle, and D. J. Kim: Sens. Actuators A 154 (2009) 103. https://doi.org/10.1016/j.sna.2009.06.007

11 R. Takei, N. Makimoto, H. Okada, T. Itoh, and T. Kobayashi: Jpn. J. Appl. Phys. 55 (2016) 1. https://doi. org/10.7567/JJAP.55.06GP14

12 T. Kanda, M. K. Kurosawa, H. Yasui, and T. Higuchi: Sens. Actuators A 89 (2001) 16. https://doi.org/10.1016/ S0924-4247(00)00530-6

13 S. P. Beeby, A. Blackbum, and N. M. White: J. Micromech. Microeng. 9 (1999) 218. https://doi. org/10.1088/0960-1317/9/3/302

14 G. Tang, J. Liu, B. Yang, J. Luo, H. Liu, Y. Li, C. Yang, D. He, V. D. Dao, K. Tanaka, and S. Sugiyama: J. Micromech. Microeng. 22 (2012) 1. https://doi.org/10.1088/0960-1317/22/6/065017

15 G. Tang, B. Yang, C. Hou, G. Li, J. Liu, X. Chen, and C. Yang: Sci. Rep. 6 (2016) 1. https://doi.org/10.1038/ srep38798

16 N. Li, Z. Yi, Y. Ma, F. Xie, Y. Huang, Y. Tian, X. Dong, Y. Liu, X. Shao, Y. Li, L. Jin, J. Liu, Z. Xu, B. Yang, and H. Zhang: ACS Nano 13 (2019) 2822. https://doi.org/10.1021/acsnano.8b08567

17 X. Dong, Z. Yi, L. Kong, Y. Tian, J. Liu, and B. Yang: J. Microelectromech. Syst. 28 (2019) 700. http://doi. org/10.1109/JMEMS.2019.2920213

18 L. Gu: Microelectron. J. 42 (2011) 277. https://doi.org/10.1016/j.mejo.2010.10.007

19 H. Liu, C. Lee, T. Kobayashi, C. J. Tay, and C. Quan: Smart Mater. Struct. 21 (2012) 1. https://doi. org/10.1088/0964-1726/21/3/035005

20 B. Yang, J. Liu, G. Tang, J. Luo, C. Yang, and Y. Li: Appl. Phys. Lett. 99 (2011) 1. https://doi. org $/ 10.1063 / 1.3664223$

21 B. Yang, C. Lee, W. Xiang, J. Xie, J. H. He, R. K. Kotlanka, S. P. Low, and H. Feng: J. Micromech. Microeng. 19 (2009) 1. https://doi.org/10.1088/0960-1317/19/3/035001

22 H. Wu, L. Tang, Y. Yang, and C. K. Soh: J. Intell. Mater. Syst. Struct. 24 (2012) 357. https://doi. org $/ 10.1177 / 1045389 X 12457254$

23 J. E. Kim and Y. Y. Kim: AIP Adv. 3 (2013) 1. https://doi.org/10.1063/1.4813314

24 K. Tao, H. Yi, L. Tang, J. Wu, P. Wang, N. Wang, L. Hu, Y. Fu, J. Miao, and H. Chang: Surf. Coat. Technol. 359 (2019) 289. https:doi.org/10.1016/j.surfcoat.2018.11.102

25 H. Xiao, X. Wang, and S. Joh: Mech. Syst. Sig. Process. 68-69 (2016) 138. https://doi.org/10.1016/ j.ymssp.2015.07.001 


\section{About the Authors}

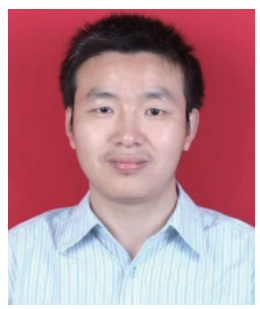

Gang Tang received his B.S. and M.S. degrees from Nanchang University, China, in 2001 and 2005, respectively, and his Ph.D. degree from Shanghai Jiaotong University, China, in 2013. Since 2017, he has been a professor at Nanchang Institute of Technology. His research interests are in MEMS, sensors, and actuators. (tanggang@nit.edu.cn)

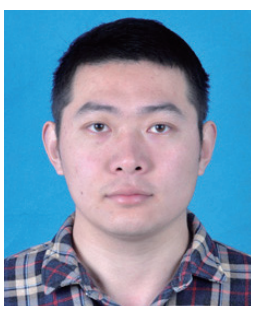

Gaoyang Xie received his B.S. degree from Wuhan University of Technology, China, in 2015 and his M.S. degree from Nanchang Institute of Technology, China, in 2018. His research interests are in MEMS, bioengineering, and sensors.(632980562@qq.com)

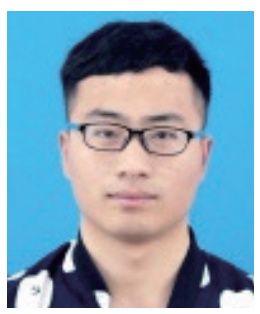

Bo Huang received his B.S. degree from Jing Gang Shan University, China, in 2018. Currently, he is studying for a master's degree in Nanchang Institute of Technology. His research interests are in MEMS and piezoelectric energy harvesting. (1399373877@qq.com.cn)

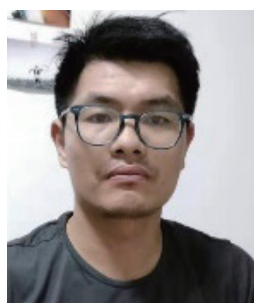

Bin Xu received his B.S. degree from Nanchang University, China, in 2005, his M.S. degree from Dalian University of Technology, China, in 2009 and Ph.D. degree from Shanghai Jiaotong University, China, in 2018. Since 2018, he has been a lecturer at Nanchang Institute of Technology. His research interests are in MEMS, sensors, and actuators. (xubin84115@163.com)

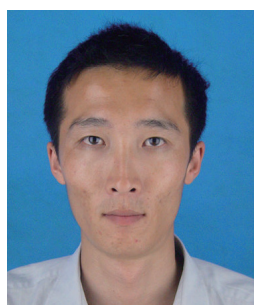

Zhibiao Li received his B.S. degree from Changchun University of Technology and his M.S. degree from Nanchang University, China, in 2003 and 2014, respectively. $\mathrm{He}$ is now a lecturer at Nanchang Institute of Technology. His research interests are in MEMS and sensors.

(lzhibiao@qq.com)

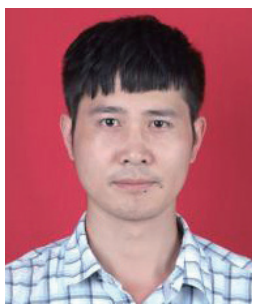

Xiaoxiao Yan received his B.S. degree from Yantai University, China, in 2005, and his M.S. degree from Nanjing University of Science and Technology, China, in 2008, and his Ph.D. degree from Shanghai Jiaotong University, China, in 2014. He is now a lecturer at Nanchang Institute of Technology. His research interests are in MEMS, Bio-MEMS, and sensors. (qwwxiao@sjtu.edu.cn) 


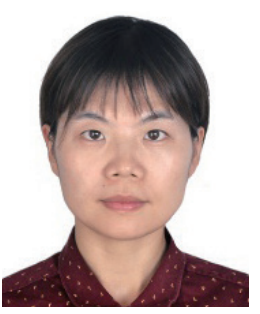

Dandan Yuan received her B.S. and M.S. degrees from Nanchang University, China, in 2009 and 2012, respectively. She is now a lecturer at Nanchang Institute of Technology. Her research interests are in MEMS, sensors and actuators. (yuandandan@nit.edu.cn)

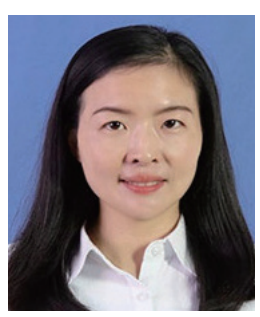

Wenjing Wu received her B.S. and M.S. degrees from Nanchang University, China, in 2004 and 2017, respectively. She is now a lecturer at Nanchang Institute of Technology. Her research interests are in MEMS and Engineering management.(283698092@qq.com) 